\title{
Thomas Bark \\ Die Entstehung des BGB im Spiegel der bürgerlichen Rechtsgeschichte
}

\author{
I. DARSTELLUNG DER ERKLARUNGSVERSUCHE*
}

\section{r. »Die Einheit des Privatrechts*}

Dieser Topos markiert eine Art der rechtshistorischen Gedankenführung, die deshalb an erster Stelle genannt zu werden verdient, weil sie formal geschlossen und in ihrer methodischen Internalisierung bestens geeignet ist, den Zustand rechtshistorischer Forschung für den Vergleich mit anderen Wissenschaften hinreichend offenzulegen. Diesen, das sei vorangeschickt, begegnet sie auch mit der selbstverständlichsten Ignoranz aller gebotenen Ansätze.

Das Gerüst von historischen Fakten, an dem sie sich oberflächlich orientiert, ist eine Abfolge von Gesetzgebungsakten, die in der Kodifikation des Bürgerlichen Gesetzbuchs ihren vorläufigen Höhepunkt und Abschluß erreichen ${ }^{1}$. Die »Einführung « von Baumann bleibt übrigens bei diesem Faktengerüst bereits stehen². - Es dient den Autoren dazu, eine Entwicklung zu materialisieren, die von der »Buntscheckigkeit der deutschen Privatrechtslandkarte $\ll^{3}$ zur gloriosen Vereinheitlichung des Privatrechts führt. Eine solche Entwicklung stand bereits den Redakteuren des BGB vor Augen, die in der Kodifizierung wesentlich die Vereinheitlichung geltenden Rechts sehen wollten, um sein Ende (zu) machen dem unleidlichen Zustand der Zerrissenheit « ${ }^{4}$. Ihre Folgerichtigkeit wird dem Leser wirksam suggeriert, indem der Anfangspunkt weit in die ehrwürdige Tradition zurückverlagert wird.

Meist ist es die Rezeption des römischen Rechts in Mitteleuropa, die in fast heroisierter Bedeutung zum schicksalhaften Anfangspunkt für diejenigen Autoren wird, die sich um die Verfolgung weitgespannter, oft mehrere Jahrhunderte umgreifender Linien bemühen: "Die Privatrechtsgeschichte ist zum größten Teil eine Geschichte des Nachlebens des römischen Rechts«(Wesenberg S. 7). Die Li-

\footnotetext{
* Der Darstellung liegt folgende Literatur zugrunde: Mitreis-Lieberich, Deutsche Rechtsgeschichte, 12. Aufl. 1971; Gerhard Wesenberg, Neuere deutsche Privatrechtsgeschichte im Rahmen der europäischen Rechtsentwicklung, 2. Aufl. bearb. v. G. Wesener, 1969; Franz. Wieacker, Privatrechtsgeschichte der Neuzert, 2. Aufl. I967.

Gustav Bohemer, Einführung in das Bürgerliche Recht, 2. Aufl. 1965; Radbruch-Zweigert, Einführung in die Rechtswissenschaft, I I. Aufl. 1964; Jürgen Baumann, Einführung in die Rechtswissenschaft, 3. Aufl. r 971 .

Paul Koschaker, Europa und das römische Recht, 3. Aufl. 1958; Georg Dahm, Deutsches Recht. Die geschichtlichen und dogmatischen Grundlagen des geltenden Rechts, I95 I, 2. Aufl. I963; Franz Wieacker, Das Sozialmodell der klassischen Privatrechtsgesetzbücher und die Entwidklung der modernen Gesellschaft. Schriftenr. d. Jur. Stud. Gesellsch., H. 3 (r953); ders., Das bürgerliche Recht im Wandel der Gesellschaftsordnungen, in: Hundert Jahre deutsches Rechtsleben. Festschr. z. hundertj. Bestehen des DJT Bd. II, r960, S. I-I 8.

1 Besonders markant Wesenberg $\$ \$ 24,25$ I, II (S. I7I f.); ähnlich Wieacker, Privatrechtsgeschichte $\$_{24}$ II (S. ${ }_{462}$ ff.), allerdings mit anspruchsvollerer Zielsetzung; Dahm S. ${ }_{4} 88 \mathrm{f}$

2 Vgl. Baumann, Einführung $\S_{3} I_{I}$

${ }^{3}$ Koschaker S. 259.

4 Mugdan, Materialien Bd. I, S. 847.
} 
nie bis hin zum BGB hat folgende typisierte Form: Als "Gemeines Recht « kommt der Corpus Iuris in einer den zeitgenössischen Bedürfnissen entsprechenden Form zur Geltung ("Usus Modernus Pandectarum») und tritt neben Gebietsrechte, wie z. B. Stadtrechte, lokale Gewohnheitsrechte oder regional geltende Landesgesetze: Die Zersplitterung des Privatrechts gilt bis zur Gründung des Deutschen Reichs I87 I als kennzeichnend. Im 19. Jahrhundert arbeitet die Historische Rechtsschule erfolgreich an der Systematisierung des römischen Rechts, eine Kontinuität, die von Savignys "System des heutigen römischen Rechts « bis zu Windscheids Pandektenlehrbuch und damit schließlich in die Vorarbeiten zum BGB hineinreicht ${ }^{5}$.

Das Überschauen derartiger Zusammenhänge scheint die Kardinaltugend des Rechtshistorikers zu sein. So schreibt Boehmer:

"Unser heutiges bürgerliches Recht ist ... nur verständlich, wenn man sich dreier großer geschichtlicher Tatsachen bewußt ist. Die erste ist die Aufnahme oder Rezeption des römischen Rechts. ... Die zweite ist... die gewaltige Geistesströmung der Aufklärung. ... Auf wissenschaftlichem Boden erwuchs aus ihr das Naturrecht. ... Es entlud sich politisch in der französischen Revolution und in der Erklärung der Menschen- und Bürgerrechte vom 28. 8. i789 und fand... gesetzlichen Niederschlag in den großen Kodifikationen der Jahrhundertwende, ... Die dritte Tatsache ist die historische Bewegung, die der Rechtsentwicklung über das sächsische BGB von I 86 s bis zur Schöpfung des deutschen Bürgerlichen Gesetzbuchs ihre Richtung gegeben hat.«(S. 56 ff.)

Ein solchermaßen am positiven Verlauf der Rechtsgeschichte orientiertes Denken vermag freilich keine Begründungen im Sinne irgendeiner Kausalität anzubieten, es sei denn, man könnte die »innere Logik « eines Prozesses dafür gelten lassen. Kennzeichnend für diesen Typus ist deshalb die Heranziehung begründender Faktoren aus anderen Bereichen des historischen Prozesses. Für die Rechtsgeschichte zum Inkraftreten des BGB ist es vor allem ein politischer Faktor, der fast als monokausal eingeführt wird: die deutsche Reichsgründung im Jahre I 87 I.

"Erst das neue Reich... konnte mit der Bürgschaft der Dauer die schmerzlich vermißte Einheit des Privatrechts herstellen: durch das BGB « (Radbruch-Zweigert S. 100).

"Die Gesetzbücher dieser Zeit sind Kinder des Bundes der bürgerlichen Gesellschaft mit dem Nationalstaat, der ... an der Wiege der Kodifikationen stand « (Wieacker PrivRG S. 46I).

An gleicher Stelle apostrophiert Wieacker die »einheitliche Staatlichkeit « als eine "Voraussetzung « der Kodifikation; Koschaker bezeichnet die Reichsgründung von I 87 I als "politische Bedingung (S. 289). Eine geistesgeschichtliche Variante dieser Analogie zum gleichzeitigen politischen Vorgang findet sich gleichfalls bei Koschaker, der die "Fortwirkung der alten Reichsidee" in der Rechtswissenschaft konstatiert.

Einheit des Privatrechts und Reichseinigung entsprechen einander bis in die Formulierungen hinein:

"(Der) Hauptzweck, den es (scil. das BGB) sich gesetzt hatte, (war) die Herstellung der deutschen Rechtseinheit « (Wesenberg S. I74).

Was von keinem der Autoren dieses Typus untersucht, ja nur problematisiert

5 Vgl. die gedrängte Darstellung bei Karl Larenz, Allgemeiner Teil des deurschen Bürgerlidhen Rechts (1967), S. 1 I. 
wird, ist das Verhältnis von Rechtsgeschichte und politischer Geschichte. Es bleibt bei einer bloßen Beschreibung der Entwicklung von der Rechtszersplitterung zur Einheit, die mit dem BGB abgeschlossen wird, so wie die Bestrebungen zur Einigung des deutschen Reichs 1871 ihr Ende finden.

\section{Die Einheit der Rechtswissenschaft *}

Auch bei einem zweiten typischen Begründungsgang wirkt die Vorstellung von der im BGB erreichten Einheit unterschwellig mit, wenn auch in einer weitaus differenzierteren und nicht so leicht plastisch präsentablen Form. Dieser Typ, der sich vor allem bei solchen Autoren findet, die Wert auf ein dogmengeschichtliches Verständnis legen (Boehmer, Koschaker, Dahm, auch Wieacker -, betrachtet das Zustandekommen einer deutschen Privatrechtskodifikation wesentlich als Leistung der deutschen Rechtswissenschaft.

Untersucht wird daher die Geschichte der Historischen Rechtsschule und die Haltung ihrer profiliertesten Vertreter zur Frage der Kodifikation. An die Stelle des Faktengerüstes der Teilrechtskodifikationen tritt hier, personenorientiert und -fixiert, der Wandel bestimmter dogmatischer Auffassungen, der als Reifungsprozeß erkennbar wird: "Die Abneigung der älteren Historischen Schule gegen die Gesetzgebung verschwindet « (Wesenberg S. I I I); ursprünglich war das Programm der Historischen Rechtsschule ein Hindernis für ein gemeindeutsches Privatrechtsgesetzbuch (Mitteis-Lieberich S. 252). Savigny, dessen bekannter Streit mit Thibaut häufig zur Einleitung dient ${ }^{6}$, bekämpfte die Kodifikation jedoch nicht als solche, sondern weil »die von ihm inaugurierte neue Richtung der deutschen Rechtswissenschaft noch nicht genügend gefestigt war, um ein neues Zivilrechtsgesetzbuch tragen zu können * (Koschaker S. 259). Koschaker, der dem Zusammenhang zwischen Juristenrecht und Kodifikation ein ganzes Kapitel widmet, identifiziert die Historische Schule mit der meinheitlichen deutschen Rechtswissenschaft «, die unter den gegebenen Umständen das zunächst Erreichbare war - womit Savigny nachträglich gerechtfertigt ist:

"Für ihn mit seiner kulturellen Reichsidee war es noch immer das Reichsrecht, das als solches zwar nicht mehr die Grundlage der Rechtseinheit, wohl aber einer einheitlichen deutschen Rechtswissenschaft für die Gegenwart hergeben konnte « (S. 263).

Und wenig später heißt es in fast programmatischer Klarheit:

"Was unter den gegebenen Umständen geleistet werden konnte, hat die historische Schule erfüllt. Es gab in Deutschland vor 1900 im Privatrecht nur Partikularrechte, zu denen auch das gemeine Recht gehörte, aber es gab nur eine Rechtswissenschaft, die gemeinrechtliche. Sie war das Werk der deutschen Pandektisten und von Savigny von vornherein als allgemeine deutsche Rechtswissenschaft gedacht. Daß jene hierbei durch die noch fortwirkende alte Reichsidee unterstützt wurden, konnten wir wahrscheinlich machen. Damit war eine Voraussetzung für die Kodifikation geschaffen: eine hoch entwickelte Rechtstheorie." (S. 289.)

Einer Rechtsgeschichte, die davon ausgeht, daß »der deutsche Professor der Rechte auf lange Zeit zum Hüter der deutschen Rechtseinheit berufen war " (Wesenberg) ${ }^{7}$, fällt es nunmehr nicht schwer, den Zeitpunkt der Kodifizierung mit einem Höhepunkt der deutschen Zivilistik gleichzusetzen, was jede weitere Er-

B Vgl. Wieacker, PrivRG S. 390 ff.; Wesenberg S. $14 \mathrm{I}$; Dahm S. 148 f.; Koschaker S. 258 f.

7 Wesenberg S. 146 ; ähnlich Koschaker S. 259. 

zwei Titeln her:

"Erstens, um in dem gegenüber dem alten Reich allerdings verkleinerten Deutschland die Rechtseinheit im Zivilrecht herzustellen, zweitens, weil das Gesetz die Ergebnisse einer hochentwickelten Rechtswissenschaft verarbeiten konnte, ohne die eine gute Kodifikation nicht denkbar ist. ${ }^{8} 8$

Hier wird das Selbstbewußtsein einer Wissenschaft beschworen, deren Entwicklung sich nach eigenen Gesetzen zu vollziehen scheint, und die darum einen Anspruch auf Autorität aus Autonomie geltend machen kann. Das rechtshistorische Denken wird der Festschreibung positiven Rechts dienstbar gemacht, wenn es gelingt, die Kontinuität rechtswissenschaftlicher Arbeit aus der Geschichte ins positive Recht hinein zu verlängern. Eben dies wird aber unter dem Thema "Einheit des Privatrechts als Höhepunkt der Rechtswissenschaft « exemplarisch vorgeführt.

Uberraschend ist dabei die Einseitigkeit, mit der die Vertreter dieser wissenschaftsgeschichtlichen Darstellungsweise operieren. Nur bei Wieacker finden sich verstreute, versteckte Hinweise darauf, daß die Pandektenwissenschaft in die Konzeption des BGB nicht nur einen überragenden dogmatischen Reflexionsstand eingebracht hat, sondern ein sehr zwiespältiges und problematisches Verhältnis zur gesellschaftlichen Wirklichkeit ihrer Zeit ${ }^{0}$. Wieacker bietet dafür eine Begründung an, die er selbst in der Nähe der Metaphysik ansiedelt: Die Philosophie Hegels, die »der Entdeckung der Geschichtlichkeit des Rechts eine ähnlich normative Bedeutung hätte geben können wie einst die naturrechtliche Anthropologie den ungeschichtlichen Rechtsentwürfen der Aufklärung «, sei ohne Einfluß geblieben - "eines der vielen Paradoxe der Geschichte des Geistes « (S. $4 \mathrm{r}_{4}$ f.). Es kann nur als absichtsvolle Vereinfachung interpretiert werden, wenn die Kontinuität von Rechtswissenschaft und Gesetzgebung, von "Juristenrecht und Kodifikation « (Koschaker) ausschließlich im Sinne normativer Pädagogik präsentiert wird, ohne daß zugleich der Zweifel historisch-kritischen Bewußtseins die längst bekannte politische Naivität der Historischen Rechtsschule ${ }^{10}$ zum bestimmenden Faktor dieses Abschnitts der Wissenschaftsgeschichte erhebt.

\section{3. "Das $B G B$ in seiner Zeit *}

Zu zwei Darstellungstypen, die sich gegenüber der allgemeinhistorischen Dimension als geschlossen erwiesen haben, tritt nun ein dritter, der den zeitgeschichtiichen Hintergrund der Kodifizierung zum Gegenstand hat. Er tritt nur bei Mitteis-Lieberich isoliert auf, die der "wirtschaftlichen und sozialen Entwicklung im 19. Jahrhundert « ein eigenes Kapitel widmen und das Inkraftreten des BGB erst später behandeln ${ }^{11}$; bei den anderen Autoren ist er assoziativ eingearbeitet ${ }^{12}$ und erlaubt Rückschlüsse auf das methodische Verhalten gegenüber den anderen Elementen des historischen Prozesses.

\footnotetext{
8 Koschaker S. 29 r f.; Wieacker spricht von 》wissenschaftlicher Spätreife «: PrivRG S. ${ }_{4}^{6 r}$.

9 Wieacker, PrivRG S. 359 (Fußn. 40), 40r, 4rs.

10 Vgl. dazu Walter Wilhelm, Zur juristischen Methodenlehre im 19. Jahrhundert. - Die Herkunft der Methode Paul Labands aus der Privatrechtswissenschaft, 1958, S. $39 \mathrm{ff}$., $80 \mathrm{ff}$. u. öfter, sowie: E. W. Bödkenförde, Die Historische Rechtsschule und die Geschichtlichkeit des Rechts, in: Stud. z. 60. Geb. v. Joachim Ritter, 1965 , insbes. S. $20 \mathrm{ff}$.

11 Mitters-Lieberich, $\oint_{4} 6$ S. 240-242; ähnlich Dahm S. 4 Is ff.

12 Vgl. beispielsweise bei Wieadker, PrivRG, die Passagen auf S. 351, 4 ro ff., 440, 442 u. ö.
} 
Wenn man erwartet, hier nun eine explizite Erklärung des Zeitpunktes der Kodifizierung zu finden, so wird man enttäuscht. Die Quintessenz aller allgemein historischen Bezüge, die in ähnlicher Form in sämtlichen untersuchten Werken wiederkehrt, ist nämlich die Feststellung, das BGB sei »schon veraltet (gewesen), als es verkündet wurde " (Wesenberg), ein Werk »wissenschaftlicher Spätreife" (Wieacker), ein "Kind des 19. Jahrhunderts « (Boehmer, Radbruch-Zweigert) oder "Spätgeborenes Kind «(Wieacker), ein »Spätes Gesetz «, das »wie es bei großen Gesetzgebungswerken der Fall zu sein pflegt, keinen Beginn, sondern den Abschluß einer bestimmten Phase unseres Rechtslebens (darstellt) « $(\mathrm{Dahm})^{13}$.

Diese Feststellungen deuten auf den unausgesprochenen folgenreichen Verzicht auf die Einordnung der Kodifikation in einen übergreifenden historischen $\mathrm{Zu}$ sammenhang bereits hin, obwohl versucht wird, diesen Kontext anklingen zu lassen und in einer Art komparativen Verfahrens an das im BGB verkörperte "Menschen- und Sozialbild « (Boehmer) heranzutragen. Das BGB markiert die Zeitenwende $z$ wischen Liberalismus und Sozialstaat, indem es einerseits die Bedürfnisse der kapitalstarken Pioniere der industriellen Revolution « (Wieacker) ${ }^{14}$ erfüllt, andererseits in bescheidenen Ansätzen soziale Erfordernisse der Zeit berücksichtigt, ohne dabei wegweisend zu sein. Daraus ergibt sich seine Rückständigkeit.

Dieses Resultat, das inhaltlich einer Verspätung der Kodifikation des deutschen Privatrechts gleichkommt, wirkt angesichts des von den Autoren tatsächlich verarbeiteten historischen Materials merkwürdig inkonsequent.

Insbesondere Boehmer liefert in seinem Buch eine vergleichsweise breite wirtschafts- und sozialgeschichtliche Situationsbeschreibung, deren konsequente Durchführung ein ganz anderes Ergebnis nahelegen würde. Sie soll etwas ausführlicher zitiert sein:

"... erwies sich gerade das Handels- und Wertpapierrecht im Laufe des 19. Jahrhunderts für das allgemeine Verkehrsrecht der bürgerlichen Gesellschaft als Pionier des rechtlichen Fortschritts und der gesetz.lichen Vereinheitlichungsbestrebungen. Diese machten sich mit der fortschreitenden Entwicklung Deutschlands vom Agrarstaat zum Industriestaat, der für gleiche wirtschaftliche Produktions- und Güterverkehrsformen auch gleiche rechtliche Normen brauchte, immer dringlicher geltend."

"Der Zeitgeist war nüchtern, materialistisch, kaufmännisch. Er stand unter dem Zeichen politischer und wirtschaftlicher Saturiertheit des... Besitzbürgertums, das... in dem neugegründeten Kaiserreich, in dem es die einstweilige Verwirklichung seines Einheitstraums erblickte, bis an die Spitze des gesellschaftlichen Ordnungsbaues vorgedrungen war und seinen Frieden mit den - wenngleich noch nicht besiegten - konservativen Kräften des preußischen Obrigkeitsstaates gemacht hatte."

"(Der Gesetzgeber) stand den schon bei Inkraftreten des Gesetzbuchs immer heftiger Gehör und Anerkennung fordernden Problemen des vermaßten gewerblichen und kulturellen Soziallebens mit seinen hochkapitalistischen Zusammenballungen, seiner technisierten Großindustrie, seinen sozialpolitischen Spannungen und Kämpfen, seinem Massenverkehr und Großstadtmilieu, ... und - last not least - den schon damals sich mit wachsender Energie anmeldenden Ansprü-

13 Wesenberg S. 174; Wieacker, PrivRG S. $46 \mathrm{r}$; Boehmer S. 83 -vgl. Radbruch-Zweigert S. 101; Wieacker, Sozialmodell S. x6; Dahm S. 490.

14 Wieacker, PrivRG S. ${ }_{4} 62$; vgl. Sozialmodell, S. 6, 10, 17; Festschrift DJT S. 6. 
chen der öffentlichen Hand auf Mitbeteiligung am Wirtschaftsprozeß, auf Len-

kung der privaten Wirtschaftstätigkeit und Beschränkung der Privatautonomie, noch einigermaßen hilf- und verständnislos gegenüber.« (S. $65,82,84$.)

Auf knappem Raum werden hier, wenn auch ohne erkennbaren inneren $\mathrm{Zu}$ sammenhang, Strukturmerkmale des gesellschaftichen und sozialen Wandels im ausgehenden 19. Jahrhundert angesprochen, die unter den Stichworten $»$ Klassenkompromiß zwischen Adel und Wirtschaftsbürgertum «"Emanzipation des Proletariats « "Konzentrationskapitalismus « und "Staatsinterventionismus « in der Tat Allgemeingut der Geschichtsschreibung dieses Zeitraums auch unter sozioökonomischem Aspekt geworden sind. Die Charakterisierung des BGB als "Kompromißgeschöpf, das den Kampf der sozial- und wirtschaftspolitischen Anschauungen der Ưbergangszeit, in der es entstanden ist, widerspiegel « $^{15}$, wirkt denn auch vergleichsweise überzeugender als die sonst übliche Betonung der sozialen Rückständigkeit des im BGB verankerten mittelständischen Wirtschaftsliberalismus.

Auch Koschaker setzt das BGB »in die Mitte einer Periode, freilich nur, um seinen konservativen Charakter von der fortschrittlichen Konzeption des Code Civile als Aufbereitung der Errungenschaften der französischen Revolution für eine neue Epoche des liberalen Bürgertums abzugrenzen ${ }^{16}$.

Das Beispiel zeigt, daß den Rechtshistorikern die wirtschafts- und sozialgeschichtlichen Zusammenhänge nicht etwa unwichtig sind; auch bei Wieacker, Mitteis-Lieberich und Radbruch-Zweigert ${ }^{17}$ finden sich inhaltlich übereinstimmende historische Vorstellungen.

Ihre Funktion ist es jedoch nicht, aus einer Analyse der gesamtgesellschaftlichen ökonomischen und politischen Entwicklung heraus den Zeitpunkt der Kodifikation erklärend zu interpretieren. Diese Hinweise dienen vielmehr allein dazu, den Hintergrund für Werturteile über das Sozialbild dieses Gesetzbuchs abzugeben $^{18}$; das ist die einzige praktische Folgerung, die die Rechtshistoriker aus der gewiß vorhandenen Einsicht in die Interdependenz von Recht und Gesellschaft zu ziehen sich genötigt sehen. Außerungen wie bei Dahm:

"Zu allen Zeiten hat das Recht eine politische Bedeutung gehabt und politischen Zwecken gedient. Jedes politische und wirtschaftliche System bildet die... seinen Zielen entgegenkommenden Rechtsformen aus. ... Der bürgerliche Kapitalismus des I9. Jahrhunderts hat sich mit dem Rechtssystem des liberalen Rechtsstaats eine Ordnung geschaffen, die seinen praktischen Zwecken in idealer Weise entsprach « (S. 178 f.), stehen weit außerhalb des Zusammenhanges und bleiben folgenlos.

\section{Metbodische Implikationen}

Die Kodifikation des Privatrechts ist ohne Zweifel für eine Wissenschaft, die von der Geschichte des Rechts handelt, von einschneidender Bedeutung. Wie ist es um sie bestellt, wenn ihr, wie zu zeigen versucht wurde, Erklärungen aus sich selbst, aus der Rechtsentwicklung heraus zu genügen scheinen; wenn das, was um

15 Boehmer, S. 84 .

16 Koachaker S. $29 \mathrm{r}$ f.; diesen Vergleich bringt auch Wieacker, PrivRG S. 479 und Sozialmodell, S. 4 .

17 Wieacker, PrivRG S. 442, 479; Sozialmodell S. 10 ff., 17; Mitteis-Lieberich S. 240 ff.; Radbruch-Zweigert S. I $00 \mathrm{f}$.

18 Wieadker, PrivRG S. 474 ff., 479; Radbruch-Zweigert S. ror ; Mitteis-Lieberich S. 252 f.; Dahm S. 490 und leider auch Boehmer S. 83 ff. 
sie her geschehen ist, nicht mehr für sie bedeuter, als eben noch erzählerischer Rahmen, Urteilskriterium oder, günstigstenfalls, Vergleichsmaßstab zu sein?

Der Umgang mit den historischen Fakten kann nicht anders als hilflos bezeichnet werden. Methodisch strukturiert ist er nicht. Diese Behauptung soll nachfolgend bewiesen werden. Sie gehört in den Zusammenhang der Frage nach dem methodischen Selbstverständnis dieser Wissenschaft, wie es aus Außerungen ihrer Autoren hervorgeht.

Die Interdependenz von Recht und Gesellschaft ist ihnen, so wurde festgestellt, in allgemeinster Form bewußt. So sie als Problem kenntlich gemacht wird, geschieht das jedoch widersprüchlich und orientierungslos. So versucht Wieacker in einem Kapitel über "Die Entdeckung der Rechtsgeschichte" die Emanzipation der Geschichte von der Dogmatik und ihre neu gefundene wissenschaftliche Zielsetzung zu beschreiben. Die Interdependenz bezeichnet er dort als »Relationalität «(!) zwischen »bestimmten rechtsgeschichtlichen Problemstellungen und -lösungen und bestimmten geschichtlichen Sozial- und Wirtschaftsstrukturen (als) ... ausreichende Vororientierung für das methodische Verfahren der Rechtshistoriker «19. In dem Wort "Vororientierung « liegt die inhaltliche Bestimmung der Interdependenz, wie an anderer Stelle sichtbar wird; die Rechtsgeschichte, so Wieacker im Vorwort seines Lehrbuchs, habe $n$ die Wirkung des rechtswissenschaftlichen Bewußtseins auf die soziale Wirklichkeit « ${ }^{20}$ zu untersuchen!

Auf welch schwankendem Boden dieses selbstbewußte Verständnis von Recht und Wirklichkeit aber steht, macht Wieacker ungewollt selbst durchsichtig, wenn er im gleichen Atemzug von "Objektivationen des sozialen Lebens gerade in Rechtsordnungen « spricht, ein Gedanke, den er an anderer Stelle in vertiefter Form verfolgt. In seinem Beitrag in der Festschrift zum roojährigen Bestehen des Deutschen Juristentags wird der "Verlust der (zivilistischen) Mitte" in der heutigen Dogmatik auf "Strukturverlagerungen der modernen Wirtschaftsgesellschaft « zurückgeführt, die in »allgemeinen dogmatischen Um- und Neubildungen $\ll$ ihren Ausdruck finden ${ }^{21}$.

Es scheint, als habe hier eine noch auf schwachen Füßen stehende Wahrheit den Rechrshistoriker verunsichert. Dieser setzt sich prompt zur Wehr: Gegen die »bewußtlose Preisgabe" des Rechts an Tendenzen der "sozialen und wirtschaftlichen Umwälzung " wird eine "Kräftigung des historischen Bewußtseins und Wille zum Wiederaufbau einer geistig und begrifflich geschlossenen Zivilrechtstheorie gesetzt $^{22}$. Diese Widersprüchlichkeit und Unsicherheit, der mit einem fast trotzigen Selbstgefühl begegnet wird, ist für die Privatrechtsgeschichte ungemein typisch. In die gleiche Richtung weisen die methodischen Außerungen in den Lehrbüchern von Wesenberg:

"Die deutsche Privatrechtsgeschichte der Neuzeit hat die Entwicklung privatrechtlicher Dogmen und privatrechtlicher Institutionen im deutschen Rechtsleben vorzutragen und die geistigen und sozialen Hintergründe dieser Erscheinungen zu erklären« (S. 7)

und, mit besonderem Pathos, Mitteis-Lieberich:

"(Die Rechtsgeschichte) zeigt, daß das Recht allezeit dem Menschen geholfen hat, sich von der blinden Naturkausalität zu befreien; so ist sie eine Freiheitslehre« (S. I).

18 Wieacker PrivRG S. 423.

20 Wieacker PrivRG S. 8. Vgl, weiter Zitate am Anfang dieses Heftes.

21 Wieacker, in: Festschrift DJT S. 6 f. u. die Gesamttendenz der Schrift zum "Sozialmodell, « insbes. S. $24 \mathrm{ff}$.

22 Wieadker, in: Festschrift DJT S. I 8 ; ebenso Sozialmodell, S. 27. 
Vor diesem Hintergrund ist nun verständlich, warum es dieser Rechtsgeschichte nur mit dem Gedanken von der Vereinheitlichung des Privatrechts (I.) und der Reife der Rechtswissenschaft (2.) gelingt, den Zeitpunkt der Kodifikation des Privatrechts zu begründen, und warum das Bewußtsein von der Reaktion des Rechts auf gesellschaftliche Entwicklungen methodisch nicht verarbeitet wird: das Selbstbewußtsein würde gestört.

Die Frage nach dem Zustand dieser Wissenschaft kann nun eine vorläufige Antwort erfahren: Eine Wissenschaft, die erzählt, "wie es wirklich geworden ist" (Joh. G. Droysen um I 850 ), hat selbst die Frage Max Webers »warum es so und nicht anders geworden ist « ( 1904 ) noch vor sich. Sie erklärt nicht, sondern beschreibt und vollzieht nach, sie begründet nicht, sondern berichtet und versteht. Die Krise des Historismus ist an ihr vorübergegangen.

\section{KONFRONTATION MIT DER GESCHICHTSWISSENSCHAFT}

Die Rechtsgeschichte hat eine wissenschaftliche Umwelt, mit der sie den Anspruch auf historisches Erklären gemein hat. Der folgende knappe Seitenblick auf die Geschichtswissenschaft soll illustrieren, wie man dort diesem Anspruch inzwischen gegenübersteht.

Seit etwa 10 Jahren ist eine intensive Methodendiskussion im Gange ${ }^{23}$, in deren Zentrum die Bedeutung der Wirtschafts- und Sozialgeschichte für Methoden und Erkenntnisziele der modernen Geschichtswissenschaft steht. Am Beginn dieser Diskussion stand 1962 die Feststellung, nun sei "der Einbruch Max. Webers in die Geschichtswissenschaft ${ }^{24}$ zu erwarten. In diesem Zusammenhang sei daran erinnert, daß bereits um die Jahrhundertwende in dem von Max Weber gegründeten Archiv für Sozialwissenschaft und Sozialpolitik die Frage einer an den sozialen Problemen orientierten Rechts-, Geschichts- und Wirtschaftswissenschaft diskutiert wurde ${ }^{25}$. - Bis vor kurzem hatte die Sozialgeschichte als Hilfswissenschaft ein Schattendasein geführt,

"dies um so mehr, als ihre Verbindung mit der ssozialen Frage، des 19. Jahrhunderts und mit kritischen Gesellschaftstheorien sie lange in die unmittelbare Nähe der Geschichte der sozialen Unterschichten, der Arbeiterklasse und der Arbeiterbewegung rückte « ${ }^{26}$.

Lange Zeit ist daher sozialgeschichtliche Forschung als marxistisch denunziert worden, und erst als man es wieder riskieren konnte, wissenschaftliche Arbeit unter Einbeziehung marxistischer Theorie zu leisten, wurde die allgemeine Di-

23 Vgl. dazu: Karl Bosl, Der soziologische Aspekt in der Geschichte, Historische Zeitschrift (HZ) 20r (1965) 6I3 ff.; Otto Brunner, Der Historiker und die Geschichte von Verfassung und Recht, HZ 209 (1969) I ff.; H. U. Wehler, Probleme der modernen deutschen Wirtschaftsgeschichte, in: Krisenherde des Kalserreichs (1970), $290 \mathrm{ff}$; ders., Theorieprobleme der modernen deutschen Wirtschaftsgeschichte, in: Festschrift für Hans Rosenberg (1970), 66 ff.; ders., Einleitung, in: Moderne deutsche Sozialgeschichte, 3. Aufl. I970, S. 9 ff,; ders., Geschichte und Soziologie (Einleitung) i972, S. I I ff.; Jürgen Kodka, Theorieprobleme der Sozial- und Wirtschaftsgeschichte, in: Geschichte und Sozıologie S. $306 \mathrm{ff}$. Begleıtende Kommentare aus der DDR in der Zeitschrift für Geschichtswissenschaft (ZfG) I6 (I968) 20s ff., I 8 (I970) 1 350 ff., I 9 (1971) $243 \mathrm{ff}$

24 E. Bayer, in: GWU I 3 ( 1962$), 358$.

$25 \mathrm{Vgl}$. den programmatischen Einleitungsbeitrag Webers in: Archiv f. Soz. Wiss. und Soz. Pol. I (I904), I ff.

26 Jürgen Kodka, in: Geschichte und Soziologie a. a. O., S. 312. 
stanz zugunsten der auch politisch inzwischen überwiegenden Konvergenzbestrebungen aufgegeben. Bei $\mathrm{H}$. U. Wehler, von dem der bisher umfassendste Versuch einer Theoriebildung auf der Basis sozialwissenschaftlicher Geschichtsforschung stammt, liest sich das wie folgt:

"Da die moderne Welt seit den Revolutionen des ausgehenden I 8. Jahrhunderts in einem historisch beispiellosen Ausmaß auf sozialökonomischen Veränderungen basiert, rücken ... Soziologie und Ơkonomie bei jeder realistischen Analyse in eine zentrale Stellung ein. Selbst wenn Politik... als Kampf um partielle und gesamtgesellschaftliche Steuerung der Herrschenden ihr eigenes Gewicht behauptet, so kann sie doch nicht losgelöst von Konjunkturzyklus und Wachstumsstörung, von Bevölkerungsvermehrung und Klassenstruktur... begriffen, erörtert, dargestellt werden. Nicht zuletzt von dieser dialektischen Verschränkung her erklärt sich die Faszination der Marx'schen Theorie, die innerhalb ihres Beziehungsgeflechts von Okonomie, Gesellschaft und Herrschaft eine schwer zu übertreffende Kraft bewiesen hat, die endogene Dynamik einer Gesellschaft, die Geschichte als Prozeß mit eigenen Antriebskräften zu begreifen. Im Rahmen einer solchen Theorie läßt sich auch der ominöse soziale Wandel inhaltlich präzisieren. $\ll^{27}$

Außerlich auslösendes Moment der Diskussion war die Entdeckung der »Industrialisierung als tiefster Zäsur der Menschheitsgeschichte seit dem Neolithikum* (Wehler) ${ }^{28}$. Nachdem die Bedeutung der Industriellen Revolution für sämtliche politischen, ökonomischen und sozialen Strukturveränderungsprozesses einmal erwiesen war - dies nicht zuletzt dank wachsender Wertschätzung der DDR-Geschichtsforschung in Westdeutschland ${ }^{29}$-, wandte man sich dem Versuch $\mathrm{zu}$, gesamtgesellschaftliche Ursachen- und Wirkungskomplexe des späten I9. Jahrhunderts systematisch offenzulegen. Die Interdependenz zwischen dem krisenhaften kapitalistischen Industriewachstum in der Phase des beginnenden Konzentrationskapitalismus und dem sozialen und politischen Wandel, begleitet von kollektiven Psychosen ideologischer Natur als Defensivstrategie konservativer Machteliten, wurde demonstriert.

Dies geschah Ende der 6oer Jahre in einer Anzahl bedeutender Monographien ${ }^{30}$; gleichzeitig wurden verstreut vorliegende Einzelschriften mit dieser methodischen Tendenz gesammelt und erneut zugänglich gemach ${ }^{31}$. Insbesondere frühe Schriften Hans Rosenbergs, der als einer der Wegbereiter der modernen deutschen Sozialgeschichte zu gelten hat, wurden überarbeitet und neu vorgestellt $\mathrm{t}^{32}$. Mit der Veröffentlichung einer umfassenden Bibliographie des sozialgeschichtlichen

27 H. U. Wehler, ebda. S. 22.

${ }^{28} \mathrm{H}$. U. Wehler, Bismarck und der Imperıalismus. 3. Aufl. Köln 1972, S. 16.

${ }^{29} \mathrm{Vgl}$. vor allem Hans Mottek (Hrsg.), Studien zur Geschichte der Industriellen Revolution. 2 Bde. Berlin 1960; ders., Wirtschaftsgeschichte Deutschlands. Bd. II, Berlin 1964; Jürgen Kuczinsky, Studien zur Geschichte des Kapitalismus, Berlin 1957; ders., Die Bewegung der deutschen Wirtschaft 1800-1946, Meisenheim 1948; Ernst Engelberg, Deutschland von I $871-1897$. Berlin 1965 .

30 Hans Rosenberg, Große Depression und Bismarckzeit. Wirtschaftsablauf, Gesellschaft und Politik in Mitteleuropa. Berlin 1967; Helmut Böhme, Prolegomena zu enner Sozial- und Wirtschaftsgeschichre Deutschlands im 19. und 20. Jahrhundert, Frankfurt 1968; ders., Deutschlands Weg zur Großmacht. 2. Aufl. Köln 1972; H. U. Wehler, Krisenherde des Kaiserreichs. Göttingen 1970; ders., Bismarck und der Imperıalismus, 3. Aufl. Köln 1972.

${ }^{31}$ Helmut Böhme (Hrsg.), Probleme der Reichsgründungszeit I 848-1879. Köln-Berlin x968; K. E. Born (Hrsg.), Moderne deutsche Wirtschaftsgeschıchte, Köln-Berlin 1967; Michael Stürmer (Hrsg.), Das kaiserliche Deutschland. Politik und Gesellschaft 1870-1918, Düsseldorf 1970; H. U. Wehler (Hrsg.) Moderne deutsche Sozralgeschichte. 3. Aufl. Köln-Berlin x 972.

${ }^{32}$ Hans Rosenberg, Der weltwirtschaftiche Struktur- und Konjunkturwandel $1848-1857$, in: H. Böhme (Hrsg.), Probleme der Reichsgründungszeit a. a. O., S. I59 ff.; Wirtschaftskonjunktur, Gesellschaft und Politik in Mitteleuropa $1873-1896$, in: H. U. Wehler (Hrsg.), Moderne 
Schriftums ${ }^{33}$ von 1789-1970 wurde deutlich, welche zentrale Rolle dieser bisherigen Aspektwissenschaft bei ihrer Integration zukommen würde.

Das Besondere an dieser Entwicklung besteht in dem hier interessierenden rechtshistorischen Rahmen darin, daß der inhaltliche Schwerpunkt der sozialgeschichtlichen Literatur exakt auf dem Zeitraum von der Industriellen Revolution bis zur Reichsgründung liegt. Die dabei behandelten Einzelfragen, wie auch die strukturierenden erkenntnisleitenden Gesichtspunkte sind für die Geschichte des Zeitraums, in dem das Privatrecht kodifiziert wurde, entschieden ein Fortschritt. Dieser läßt sich dahin kennzeichnen, daß der historische Prozeß nunmehr bewußt als von der Entwicklung der Produktivkräfte getragen angesprochen wird; dies ist ein Schritt auf dem Wege, soziale, politische oder eben auch rechtliche Einzelphänomene im übergreifenden Zusammenhang der Entwicklung der kapitalistischen Produktionsweise zu erfassen. Freilich wird dieser Schritt weniger entschlossen als in der marxistischen Geschichtsforschung getan ${ }^{34}$. Dies berücksichtigt, lassen sich in etwa drei begründende Elemente angeben:

Zum einen ist im Zeitalter wachsender Bedeutung der Wissenschaften für den Produktions- und Reproduktionsprozeß der Gesellschaft der Zwang zur interdisziplinären Kooperation offenbar geworden. Daraus folgt die Notwendigkeit der Bemühungen, der Geschichtstheorie des Historischen Materialismus etwas entgegenzusetzen, das wie jene dem Anspruch gerecht werden kann, Beitrag zu einer umfassenden Wissenschaft von der Gesellschaft zu sein.

Zum zweiten hat der Abwehrkampf der Historie um ihren Platz in der Schulausbildung gegen die neu entstandene politische Wissenschaft ein Stadium erreicht, in dem selbst ihren konservativsten Vertretern die Dringlichkeit überzeugender theoretischer Aussagen zum Bewußtsein kommt ${ }^{35}$. Die landläufig beklagte »Geschichtslosigkeit unserer Zeit« legt dafür beredtes Zeugnis ab. Drittens ist die Renaissance der sozialgeschichtlichen Forschung als Antwort auf die Herausforderung gesellschaftswissenschaftlicher, insbesondere soziologischer Theoriebildung zu sehen; soziologische Ansätze der Befriedung sozialer Konflikte gewinnen zunehmend an Bedeutung.

Spätestens an dieser Stelle wird unübersehbar deutlich, daß der neuen Richtung der Geschichtswissenschaft etwas Dezisionistisches, Zufälliges anhaftet. Alle drei genannten Gründe sind nicht methodisch-theoretischer, sondern reaktiv-praxisbezogener Natur. Auch hier tritt wieder der Unterschied zu einem erklärt dialektischen Geschichtsverständnis zutage, bei dem der Prozeß der Theoriebildung selbst Ausdruck der Identität von Gesellschaft und gesellschaftlicher Bewußtwerdung ist. Demgegenüber ist die Theoriebildung der modernen deutschen Sozialgeschichte der Geschichte äußerlich. ${ }^{36}$

Mit diesen Andeutungen, welche Auseinandersetzung der Privatrechtsgeschichte noch bevorsteht, muß es die vorliegende Untersuchung bewenden lassen. Ihre Einschätzung läuft schließlich darauf hinaus, daß eine sozialwissenschaftliche Erneuerung der Privatrechtsgeschichte ihrerseits erhebliche Probleme aufwirf, wenn man berücksichtigt, daß komplexe Forschung stets ihre Ergebnisse auf die Funktionalität für die Gesellschaft bzw. deren herrschende Interessen zu reflek-

\footnotetext{
deutsche Sozialgeschichte a. a. O., S. 225 ff.; vgl. bereits den Aufsatz "Political and Social Consequences of the Great Depression in Central Europe $1873-1896 \%$ in: Economic History Review, Jahrg. 1943.

33 In: Moderne deutsche Sozialgeschichte a. a. O., S. 565-597; erstmals in der I. Aufl. 1967.

3 (Fn. I I), Io. Aufl. r 966, S. 234

$35 \mathrm{Vgl}$. die oben Fn. 29 angegebene Fachliteratur aus der DDR.

38 Vgl. die Nachweise in dem provozierenden Diskussıonsbeitrag von Reinhard Kosellek, Wozu noch Historie? $\mathrm{HZ} 2$ r2 (1971) r ff.
} 
tieren hat. Diese Funktionalität ist aber desto wirksamer gegeben, je umfassender gesellschaftswissenschaftliche Arbeit als Ideologie verfügbar wird.

Die Isolation der Privatrechtsgeschichte in ihrem gegenwärtigen Zustand wird an ihrer Befassung mit dem Inkraftreten des BGB beispielhaft deutlich. Sie steht in der Tradition einer fast archivarisch zu nennenden Geschichtsschreibung, die die rechtlichen Zusammenhänge isoliert und in der politische und soziale Aspekte Fremdkörper bleiben. Ihnen gegenüber ist es das Verfahren einer klassischen Spezialwissenschaft, geschichtliche Wirkungszusammenhänge rein illustrativ zu verwenden, zum Beispiel um das im BGB enthaltene Gesellschaftsmodell bewerten zu können. Das Ergebnis des schlichten Vergleichs, der mit der historischen Wirklichkeit angestellt wird, ist dementsprechend nichtssagend: Das BGB ist Ausdruck des Sozialmodells der kleinwarenproduzierenden Wirtschaftsgesellschaft, deren Träger, gewerblicher Mittelstand und Landwirtschaft, begünstigt werden. Darüber hinaus reicht es eben noch zur epochalen Kennzeichnung des Gesetzwerks: Es gehört dem Liberalismus an und ist vom "Tropfen sozialen Ols» (Otto v. Gierke) sparsam gesalbt.

\section{SKIZZE EINES MATERIALISTISCHEN ERKLARUNGSANSATZES}

Eine rechtshistorische Erklärung für den Zeitpunkt der Entstehung des BGB auf der Grundlage sozialgeschichtlicher Forschung hat von einem materialistischen Verständnis der gesellschaftlichen Funktion des Rechts auszugehen: Das Recht reguliert auf dem Boden einer bestimmten ökonomischen und gesellschaftlichen Formation die den jeweiligen Eigentumsverhätlnissen adäquaten sozialen Beziehungen zwischen den Gliedern der Gesellschaft. Recht ist daher Ausdruck der Entwicklung der zugrundeliegenden Formation und in dieser programmiert.

Ausgangspunkt ist als objektiver historischer Prozeß des 19. Jahrhunderts die Entwicklung der kapitalistischen Produktionsweise.

Sie wird zu Beginn des Jahrhunderts eingeleitet durch die Ablösung des Feudalismus. Da es in Preußen weder eine starke gewerbliche Bourgeoisie, noch kapitalistische Produktionsverhältnisse auf dem Lande, noch einen einheitlichen nationalen Markt gab ${ }^{37}$, wurden die bürgerlichen antifeudalen Tendenzen erst relativ spät artikuliert. Der preußische Staat begegnete ihnen durch eine "Reform von oben « ${ }^{38}$, die im Wege eines Kompromisses zwischen Adel und Bürgertum zur allmählichen Angleichung der Stände führte: I 807 wird die Freiverkäuflichkeit der Rittergüter eingeführt; dem Adel, der infolge der Kriegskontributionen und wegen der mangelnden Ausnutzung agrarischer Produktivität verarmt ist ${ }^{39}$, wird der Zugang zu bürgerlichen Berufen eröffnet. Mit der Entstehung der Großgrundbesitzerschicht wachsen Adel und kapitalkrättiges Bürgertum zusammen. Gleichzeitig wird als Folge der Bauernbefreiung eine breite ländliche Unterschicht freigesetzt, die zunehmend proletarisiert und als industrielle Reservearmee verfügbar ist.

\footnotetext{
${ }^{37}$ Helmut Böhme, Prolegomena zu einer Sozial- und Wirtschaftsgeschichte Deutschlands a. a. O., S. $22 \mathrm{f}$.

39 Hans Mottek, Wirtschaftsgeschichte a. a. O., Bd. II. S. $76 \mathrm{f}$.

39 "Die Produktivitätsgrenze wird bestimmt durch die Produktionstechniken« W. Rostow, zit. bei Böhme a. a. O., S. I 8.
} 
Wendepunkt in der gesamtgesellschaftlichen Entwicklung des 19 . Jahrhunderts ist die Revolution von 1848 .

Obwohl es nicht zu einer Ablösung des Obrigkeitsstaates durch eine liberale Verfassung kam, wurden zumindest die für das Kapital unumgänglichen Minimalkonzessionen durchgesetzt, begünstigt durch die Agrarkrise von I $845 / 46^{40}$. Ein Ministerium für Handel, Gewerbe und öffentliche Arbeiten wurde eingerichtet; das Abgeordnetenhaus erhielt das Budget- und Steuerbewilligungsrecht. Zwar hatte das Bürgertum trotz wachsender ökonomischer Potenz noch keineswegs die adäquate politische Repräsentation erreicht. Dennoch setzte die Revolution von I 848 im Zuge der politischen Entspannung ökonomische Kräfte frei, die früher noch in die rückständige obrigkeitliche Staatsverfassung und die stagnierende agrarische Wirtschaftsformation eingebunden waren. Die Kapitalbewegung nimmt nunmehr einen nie gekannten Aufschwung ${ }^{41}$, während das Bürgertum in den 3 oer Jahren aus Furcht vor Kapitalverlust und Anarchie sein flüssiges Kapital noch gehortet oder ins Ausland transferiert hatte. Dieses kommerzielle Kapital wird nun in großem Umfang in produktives Industriekapital der politisch nicht länger verpflichteten neuen Unternehmerschicht aus Bürgern und Großgrundbesitzern umgewandelt; die absolute Erhöhung des Kapitals wird durch die Goldfunde in Kalifornien und Australien begünstigt. ${ }^{42}$ Gleichzeitig findet eine revolutionierende Neuordnung des Gütermarktes statt. Dank der Fortschritte im Eisenbahn- und Schiffbau wird das Transportwesen derart vervollkommnet, daß schon bald die regionalen Märkte unter dem Dach des Zollvereins zu einem einheitlichen Binnenmarkt, einem geschlossenen Wirtschaftsgebiet verschmelzen, dessen Industrialisierung jetzt fast zwangsläufig ist. ${ }^{43}$ Die gewaltigen Aufgaben, die sich dem ehemals kommerziellen Kapital bei der Entwicklung der Schwerindustrie stellen, werden mit Hilfe neuer Kapitalbeschaffungsmethoden bewältigt. Einer geschickten Bankpolitik gelingt es, durch Dezentralisierung des Kredit- und Depositenwesens die Freizügigkeit der Aktie zu erreichen, ein Vorgang, der schließlich die Regierung zwingt, die Zulassungspflicht für Aktiengesellschaften aufzuheben. Die anonyme Unternehmensform ist der Höhepunkt in der Reihe der kredit- und emissionspolitischen Maßnahmen, die in Gestalt des »Effektenkapitalismus die Finanzierung neuer Großindustrien und die Erschließung neuer Märkte sichern:

"Die 18 goer Jahre sind die wichtigste spekulative Periode, die Deutschland bisher erlebt hat. In ihnen wird der moderne Kapitalismus definitiv zur Grundlage der Volkswirtschaft gemacht. «14

Diese Phase erreicht ihren vorläufigen Abschluß im Milliardenboom r 87x-73 ("Gründerjahre"). Er beschließt auch vorläufig die ökonomische Durchsetzung des Bürgertums.

Unmittelbar darauf wendet sich die Konjunktur zur ersten zyklischen Krise des modernen deutschen Kapitalismus ${ }^{45}$. In ihr bahnt sich die endgültige wirt-

40 Näher dazu Hans Rosenberg, Der weltwirtschaftliche Struktur- und Konjunkturwandel 1848 bis 1857 , in: H. Böhme (Hrsg.), Probleme der Reichsgründungszeit a. a. O., S. r60; Mottek, Wirtschaftsgeschichte a. a. O., S. is $\mathrm{f}$.

41 Rosenberg a. a. O., S. I62; vgl. auch Mottek, Studien zur Geschichte der Industriellen Revolution a. a. O., Bd. I, S. 34 f.

42 Mottek, Studien a. a. O., S. $38 \mathrm{ff}$.; Rosenberg a. a. O., S. 159.

43 Hans Haussherr, Der Zollverein und die Industrialisierung, in: K. E. Born (Hrsg.), Moderne deutsche Wirtschaftsgeschichte a. a. O., S. 57; Mottek, Studien a. a. O., S. 49 f.

44 Werner Sombart, Deutsche Volkswirtschaft im 19. Jahrhundert. 1903, S. 95; vgl. auch Rosenberg a. a. O., S. I 80.

43 Zur Krise und Depression der joer Jahre vgl. Rosenberg, Wirtschaftskonjunktur, Gesellschaft und Politik in Mitteleuropa 1873-1896, in: H. U. Wehler (Hrsg.), Moderne deutsche Sozial- 
schaftspolitische Umstrukturierung Deutschlands an. Die Industrielöhne übersteigen im Jahre 1878 erstmals die Agrarlöhne. In der sogenannten Binnenwanderung von Ostdeutschland ins Ruhrgebiet und der Verstädterung verliert der Agrarsektor zunehmend an Einfluß. Die Bevölkerungsumschichtung und die Pluralisierung des Berufsraums ${ }^{46}$ zerstören die Machtbasis des konservativen Altpreußentums, der "Rittergutsbesitzerklasse (Rosenberg). Da gleichzeitig der revolutionäre Druck der Arbeitermassen immer massiver wird, tritt das $» D i-$ lemma des industrialisierten Agrarstaates ${ }^{47}$ offen zutage.

Es besteht darin, daß ein feudaler Obrigkeitsstaat die Aufgaben nicht erfüllen kann, die eine kapitalistische Organisation der Gesellschaft an ihn stellt. Die politische Vormachtstellung der junkerlich-konservativen Kräfte, die auf der Sonderstellung des privaten Großgrundeigentums innerhalb der bürgerlichen Verkehrs- und Konkurrenzgesetze beharren, ist ein wirksames politisches Hindernis der vom Bürgertum erstrebten Kapitalisierung der Gesellschaft, ihrer vollständigen Unterwerfung unter die Gesetze des entfalteten Warenverkehrs. Dieser Widerspruch bestimmt die Kodifikationsgeschichte des Zivilrechts bis zur Vereinheitlichung im BGB.

War die Verankerung der bürgerlichen individuellen Eigentums- und Freiheitsrechte in den 1848 und danach erlassenen Verfassungen der Einzelstaaten noch eine wesentliche Unterstützung des industriellen Aufschwungs gewesen, so hatte das Bürgertum weder 1848 noch bei der Reichsgründung $1871^{48}$ politische öffentliche Rechte erhalten; vielmehr konnte es nur über die Gesetzgebung in den Parlamenten und später im Reichstag politisch mitbestimmen. Daher ließen sich zunächst nur die den dringendsten Bedürfnissen des Warenverkehrs und der rechtlichen Organisation der Kaufleute entsprechenden Teilkodifikationen durchsetzen: die Wechselordnung von 1848 und das Handelsgesetzbuch vom 3r. 5 . I 86I, die für die Vereinheitlichung des Wirtschaftsgebietes unumgänglich waren. Darüber hinaus kamen weitere Fortschritte in Richtung auf eine einheitliche Gesetzgebung nur gegen den zähen Widerstand der politisch dominierenden konservativen Kräfte zustande, die sich auf Art. 4, Ziff. I 3 der Reichsverfassung vom 16. 4. I 87 I stützen konnten. Die Anstrengungen der Nationalliberalen Partei als parlamentarischer Vertretung des Wirtschaftsbürgertums gewannen erst am 20. I 2. I873 ("Lex Lasker") Aussicht auf Erfolg. Der Weg zur Schaffung eines Privatrechtsgesetzbuchs war frei.

Die vorstehende Skizze läßt sich dahin zusammenfassen, daß es in letzter Instanz der Zusammenbruch der agrarischen Wirtschaftsformation als Machtbasis der obrigkeitlich-feudalen Staatsorganisation unter dem Druck der Industrialisierung war, der die Entstehung des BGB ermöglichte. Erst jetzt war es möglich geworden, sämtliche bürgerlichen Lebensbereiche den Verkehrsgesetzen der kapitalistischen Ókonomie zu unterwerfen. Produktion, Verteilung und Verkehr der Produkte können jetzt nach Regeln gefaßt werden, die die geltenden Normalbedingungen kapitalistischer Reproduktion juristisch verankern.

Die hier nur angedeutete materialistische Rechtsgeschichte zum Inkraftreten des

geschıchte a. a. O., S. 232 ff., 285 ff.; K. E. Born, Sozialer und wirtschaftlicher Strukturwandel Deurschlands am Ende des 19. Jahrhunderts, in: H. U. Wehler a. a. O., S. 271 ff.; Böhme, Prolegomena a. a. O., S. 84 ff.

46 Vgl. dazu Otto Neuloh, Sozialer Wandel und Industrialiserung im 19. Jahrhunderts - Versuch eines Ordnungsschemas, in: W. Rüegg, O. Neuloh (Hrsg.), Zur soziologischen Theorie und Analyse des 19. Jahrhunderts, Göttingen 1971, S. $65-80$.

47 Böhme, Prolegomena a. a. O., S. $8 \mathrm{I}$.

${ }^{48} \mathrm{Zu}$ den Problemen der Reichsgründung vgl. insbesondere Wolfgang Zorn, Wirtschafts- und sozialgeschichtliche Zusammenhänge der deutschen Reichsgründungszeit I850-1879, in: H. Böhme (Hrsg.), Probleme der Reichsgründungszeit a. a. O., S. 296-316. 
BGB kann ein Doppeltes leisten. Sie kann einmal die Entstehung privatrecht-

licher Institutionen als normative Fixierung kapitalistischer Verkehrsformen sichtbar machen, die die Anforderungen der kapitalistischen Produktionsweise an eine Kalkulierbarkeit der Profitchancen erfüllt. Sie kann zum anderen den Anstoß geben zur Reflexion auf das Verhältnis der Gesellschaft zum Recht im Sinne einer sozial und politisch verfügbaren Wirklichkeit. Als solche sollte das Recht auch in der Ausbildung ins Bewußtsein treten.

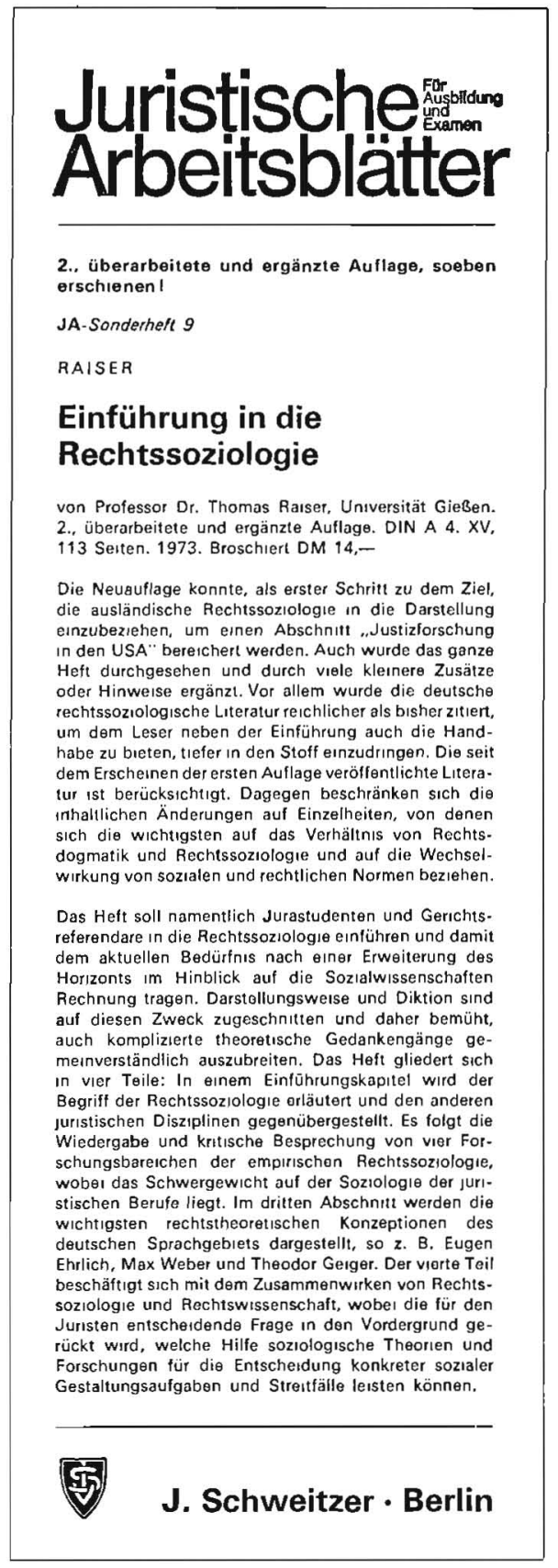

